\title{
阿秒脉冲产生的技术原理及进展
}

魏志义 ${ }^{1,2,3^{*}}$, 许思源 ${ }^{1,4}$, 江昱佼 ${ }^{1,4}$, 高亦谈 ${ }^{1,2}$, 赵昆 $^{1,3^{*}}$, 朱江峰 $^{4}$

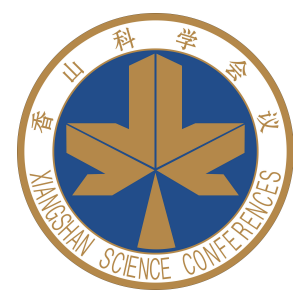

1. 中国科学院物理研究所, 北京凝聚态物理国家研究中心, 北京 100190;

2. 中国科学院大学, 北京 100049 ;

3. 松山湖材料实验室, 东莞 523808 ;

4. 西安电子科技大学物理与光电工程学院, 西安 710071

*联系人, E-mail: zywei@iphy.ac.cn; zhaokun@iphy.ac.cn

2020-11-27 收稿, 2021-01-18 修回, 2021-01-18 接受, 2021-01-19 网络版发表

国家重点研发计划(2018YFB110720，2017YFB0405202)、国家自然科学基金(61690221，91850209，11774277)、中国科学院仪器研制项目 (YZ201658)和中国科学院前沿科学重点研究计划(QYZDJ-SSW-JSC006)资助

摘要随着超短脉冲激光技术的快速发展, 人们观察超快动力学的视野从飞秒领域跨入到阿秒领域. 由于电子绕 氢原子核转 1 圈的时间大约为 $1.5 \times 10^{-16} \mathrm{~s}$, 即 $150 \mathrm{as}$, 因此阿秒激光脉冲的出现为人类提供了打开原子内部动态世界 大门的钥题, 成为 21 世纪激光物理与技术最重要的进展之一. 经过多年的不断创新和突破, 阿秒脉冲产生技术从最 初只能用少周期飞秒脉冲作为驱动光源, 已发展出多种空间及时间选通技术, 所能产生的最短激光脉宽已达43 as. 本文通过总结这些技术方法, 介绍了产生阿秒激光脉冲的原理及主要进展.

关键词阿秒脉冲, 阿秒选通技术, 高次谐波, 阿秒脉冲压缩

对瞬态物理现象的观测和研究, 是人类认识自然 现象和探索科学规律的重要主题之一. 因此, 高时间分 辨率的技术手段一直吸引着人们的研究兴趣. 在脉冲 激光出现之前, 人们利用高速相机所能达到的时间分 辨在微秒 $\left(1 \mu \mathrm{s}=10^{-6} \mathrm{~s}\right)$ 甚至纳秒 $\left(1 \mathrm{~ns}=10^{-9} \mathrm{~s}\right)$ 量级, 尽管 在导弹发射、核爆等工程技术中发挥了重要作用，但 不足以探测特征时间更短的分子尺度的微观运动过程. 想要观测分子的转动和振动过程, 需要皮秒 $(1 \mathrm{ps}=$ $\left.10^{-12} \mathrm{~s}\right)$ 和飞秒 $\left(1 \mathrm{fs}=10^{-15} \mathrm{~s}\right)$ 量级的时间分辨. 由于氢原 子中电子的玻尔半径约为 $5.3 \times 10^{-11} \mathrm{~m}$, 其绕原子核转 1 圈需要的时间约为 $1.5 \times 10^{-16} \mathrm{~s}$, 即 150 as(阿秒， attosecond, $1 \mathrm{as}=10^{-18} \mathrm{~s}$ ), 因此观察电子甚至原子核内的运动 过程, 需要时间分辨率进一步达到阿秒甚至入秒(zeptosecond, $1 \mathrm{zs}=10^{-21} \mathrm{~s}$ ) 尺度.

激光是目前人们获得高时间分辨能力最主要甚至
唯一的工具. 自 1960年Maiman ${ }^{[1]}$ 发明激光以来, 经过调 $\mathrm{Q}$ 及锁模技术的发展, 激光所能产生的脉冲宽度已从纳 秒、皮秒发展到飞秒量级, 并从科研应用到工业、生 物、医学、成像等领域. 目前, 利用先进的锁模及脉冲 放大压缩等先进技术，人类在可见及近红外波段已能 产生小于 $10 \mathrm{fs}$ 的激光脉冲 ${ }^{[2 \sim 5]}$; 通过超连续光谱展宽, 可支持短至 $2.6 \mathrm{fs}$ 的周期量级脉冲 ${ }^{[6]}$. 但要进一步获得 更短的阿秒脉冲，传统的锁模及脉冲压缩技术已经无 能为力. 虽然最近几年, 人们利用相干合成技术得到了 数百阿秒的结果, 但严格来讲, 这还只是亚飞秒脉冲 ${ }^{[7]}$.

随着激光脉冲能量的提高, 经过光学聚焦, 当焦点 处光强达到 $10^{12} \mathrm{~W} / \mathrm{c} \mathrm{m}^{2}$ 时, 其对应的电场峰值 $\left(2.7 \times 10^{9} \mathrm{~V} / \mathrm{m}\right)$ 就与原子内部束缚电子的库仑场 $\left(10^{9} \mathrm{~V} / \mathrm{m}\right.$ 量级)相当了. 此时, 将气体靶置于激光焦点附 近，靶内原子中的电子就可以从束缚态电离进人自由

引用格式: 魏志义, 许思源, 江昱佼, 等. 阿秒脉冲产生的技术原理及进展. 科学通报, 2021, 66: 889-901 Wei Z Y, Xu S Y, Jiang Y J, et al. Principle and progress of attosecond pulse generation (in Chinese). Chin Sci Bull, 2021, 66: 889-901, doi: 10.1360/TB2020-1525 
态. 早在1963年, 红宝石激光器就被应用于气体电离的 实验 ${ }^{[8,9]}$. 1965年, Keldysh ${ }^{[10]}$ 提出了光场中隧穿电离 (tunnel ionization)的理论. 1979年, Agostini等人 ${ }^{[11]}$ 观察 到了阈上电离(above-threshold ionization, ATI)现象, 阈 上电离产生的光电子能量可达到几倍至几十倍激光光 子的能量. 强场电离(strong-field ionization)这一研究领 域也就逐渐成形了. 20 世纪 80 年代, 人们观察到伴随强 场电离的高次谐波产生(high-order harmonic generation, $\mathrm{HHG}$ )的光子辐射 ${ }^{[12,13]}$, 其光谱是梳齿形状的尖峰(谐 波), 谐波的光子能量也是激光光子能量的几倍至几 十倍.

1987年, Kuchiev ${ }^{[14]}$ 在理论上提出激光场中电子电 离后二次散射的两步模型, 用来解释ATI产生的高能量 光电子. 1993年, Schafer等人 ${ }^{[15]}$ 也提出了多次散射的两 步模型, 包括初始电离和电子在激光场中的加速. 由于 激光场是交变电场, 当电场方向改变时, 电子有可能飞 回到离子附近. 同年, Corkum ${ }^{[16]}$ 详细阐述了强场电离 的半经典三步模型, 头两步是电离和电子在激光场中 的加速, 电子有可能获得很高能量. 根据第三步电子回 到离子附近时的不同物理过程, 解释了强场电离中的 3 种现象: 双电子电离、阈上电离、电子与离子复合成 原子. 在复合过程中, 电子携带的能量变成一个光子释 放出来, 即高次谐波光子. 当飞秒激光轰击气体原子, 该电离-加速-复合的三步过程在激光的每半个光周期 中发生一次，大量参与反应的原子辐射出的高次谐波 光子形成一个光脉冲. 由于HHG的中心波长可延伸到 极紫外(extreme ultra-violet, XUV)乃至X射线波段, 其 光场振荡周期在阿秒量级, 只要有足够的光谱宽度就 能够形成阿秒脉冲. 通常使用的飞秒激光脉冲包含几 个到几十个光周期，会产生几个到几十个这样的阿秒 脉冲, 并由于光谱干涉形成梳齿状的高次谐波光谱. 三 步模型为这一系列现象勾画出了一个完整直观的物理 图像, 成为高次谐波和阿秒光学的理论基础. 基于此, 1994年, Corkum等人 ${ }^{[17,18]}$ 又利用量子理论对HHG进行 了细致的描述, 并预言了单个或称“孤立”阿秒脉冲(isolated attosecond pulse, IAP, 区别于HHG的阿秒脉冲序 列)产生的理论和方法. 由于在瞬态测量中, 高次谐波难 以提供阿秒精度的时间零点和测量误差, 人们在实验 中采用多种选通方法从 $H H G$ 中获得孤立阿秒脉冲 ${ }^{[19,20]}$.

2001 年, Paul等人 ${ }^{[21]}$ 利用 $40 \mathrm{fs}$ 的钛宝石激光轰击氩 气产生13 19阶的高次谐波，测量了单脉冲宽度为 250 as的脉冲串. 同年, Hentschel等人 ${ }^{[22]}$ 利用 $7 \mathrm{fs}$ 激光脉冲
在㲵气中产生高次谐波并选出光子能量 $90 \mathrm{eV}$ 左右(波 长约 $14 \mathrm{~nm}$ )的一段光谱, 测量结果显示这是一个大约 650 as的脉冲，是第一个孤立阿秒脉冲的实验测量结 果. 2004年, Kienberger等人 ${ }^{[23]}$ 利用 5 fs激光结合阿秒条 纹相机 ${ }^{[24]}$ 测量了脉宽为 250 as的孤立脉冲. 2006年, Sansone等人 ${ }^{[25]}$ 将脉宽进一步缩短到 130 as. 2008年, Goulielmakis等人 ${ }^{[26]}$ 获得了 80 as的孤立脉冲. 2012年, Zhao等人 ${ }^{[27]}$ 获得了 67 as的孤立脉冲, 中心光子能量为 $90 \mathrm{eV}$, 这是至今为止以钛宝石激光器为驱动光源获得 的最短阿秒脉冲. 由于阿秒激光给超快科学发展带来 的重要意义, 国内也开展了阿秒激光脉冲相关的研究, 并取得了系列有重要影响的理论与实验进展. 2013年, 中国科学院物理研究所实现了160 as孤立阿秒脉冲测 量实验结果 ${ }^{[28]}$, 标志着我国阿秒激光研究的重要突破. 2017 年，Li等人 ${ }^{[29]}$ 使用脉宽 $12 \mathrm{fs}$ 、中心波长 $1.7 \mu \mathrm{m}$ 的 红外激光作为驱动光源, 获得了 53 as的孤立脉冲, 中 心光子能量为 $170 \mathrm{eV}$ (波长 $7.3 \mathrm{~nm})$, 第一次将阿秒脉冲

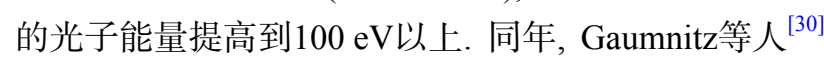
使用类似的驱动光源在中心光子能量 $100 \mathrm{eV}$ 附近获得 43 as的脉冲, 成为最新的相干光脉冲世界纪录. 上述一 系列成果(图1), 标志着超快科学与技术迎来了阿秒时 代, 并由此掀起了阿秒激光产生与应用的热潮, 成为超 快科学最前沿的研究热点 ${ }^{[19,20,31]}$. 2020年, 华中科技大 学 ${ }^{[32]}$ 、国防科技大学 ${ }^{[33]}$ 和中国科学院西安光学精密机 械研究所 ${ }^{[34]}$ 的研究团队也先后实现了阿秒激光脉冲的 产生和测量. 这些重要进展为人们利用阿秒激光开展 原子内部电子动力学的研究，揭示发生在微观世界瞬

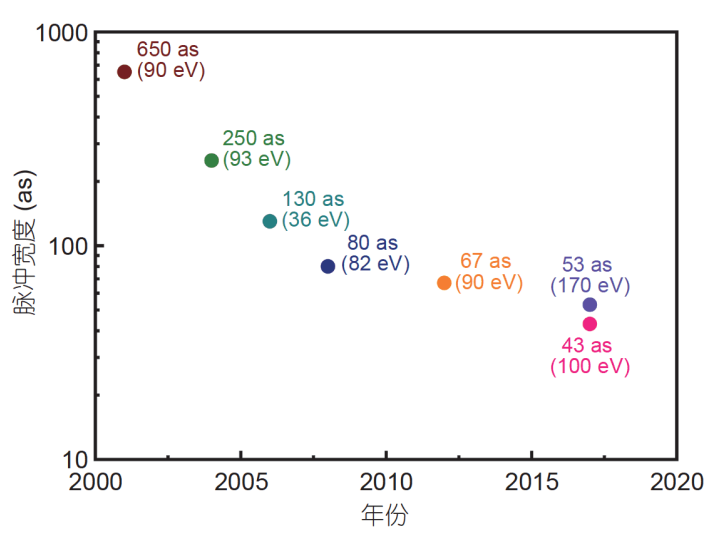

图 1 (网络版彩色)最短孤立阿秒脉冲的发展历史. 括号中参数为中 心光子能量

Figure 1 (Color online) Evolution of the world record for isolated attosecond pulse width. Numbers in the brackets are the central photon energies 
态过程中的科学问题提供了前所未有的机遇.

对于已经产生的孤立阿秒脉冲或者脉冲串，如何 对其脉宽和相位等参数进行测量也是一个关键问 题 ${ }^{[19,20,31]}$, 目前主要是利用阿秒脉冲(或脉冲串)电离气 体原子获得光电子并测量其能谱, 再叠加一个飞秒脉 冲于作用区，对所产生的光电子能谱进行调制，相当于 一个阿秒与飞秒脉冲的互相关过程. 调节阿秒与飞秒 脉冲之间的相对延时可以得到带有调制的二维光电子 能谱图，再经过反演计算提取出阿秒脉冲或脉冲串的 脉宽等信息. 较为常用的方法包括测量孤立阿秒脉冲 的阿秒条纹相机 (attosecond streak camera) ${ }^{[24]}$ 和测量阿 秒脉冲串的双光子干涉阿秒拍频重建(reconstruction of attosecond beating by interference of two-photon transitions, RABITT $)^{[35]}$ 方案. 受篇幅所限, 将另外撰文介绍 阿秒测量 ${ }^{[36]}$.

基于阿秒脉冲的上述重要进展，本文将介绍阿秒 激光形成的原理及发展中的主要技术突破，重点介绍 孤立阿秒脉冲的产生和目前获得孤立脉冲的各种选通 技术。

\section{1 高次谐波产生原理}

三步模型给出了 $\mathrm{HHG}$ 与传统非线性光学中谐波产 生原理不同的半经典物理图像 ${ }^{[16]}$, 具体的物理过程如 下(图2).

第一步, 在线偏振飞秒激光电场的作用下, 原子的 库仑势开始倾斜, 导致电子逃离的门槛降低, 并最终发 生隧穿电离. 第二步, 电离后电子在激光电场的作用下 飞离原子，当光场变为 0 并反向后，电子减速并最终改 变飞行方向, 有可能加速飞回原子. 第三步, 飞回原子 附近的电子与原子相互作用, 有可能发生以下 3 种物理 过程之一: 非弹性散射、弹性散射、与原子复合. 前两 种是与原子内其他电子之间的散射过程：非弹性散射 将导致双电子电离; 弹性散射的结果即为ATI. 第三种 是当飞回的电子与原子复合并回到基态时，其在光场 中获得的动能与从连续态到基态的跃迁能(等于电离 能)之和以高次谐波光子的形式辐射出来. 电子在激光 场不同时刻电离后, 在光场中飞行获得的能量不同, 最 终复合时释放的能量也不同，因此产生覆盖较宽的光 谱, 形成一个极紫外脉冲. 该三步过程在飞秒驱动激光 每半个周期发生一次，形成一个等时间间距的极紫外 脉冲序列，并在频域发生干涉而形成梳齿状的分立 HHG光谱(图3). 按照谐波级次的强弱变化分类，其频

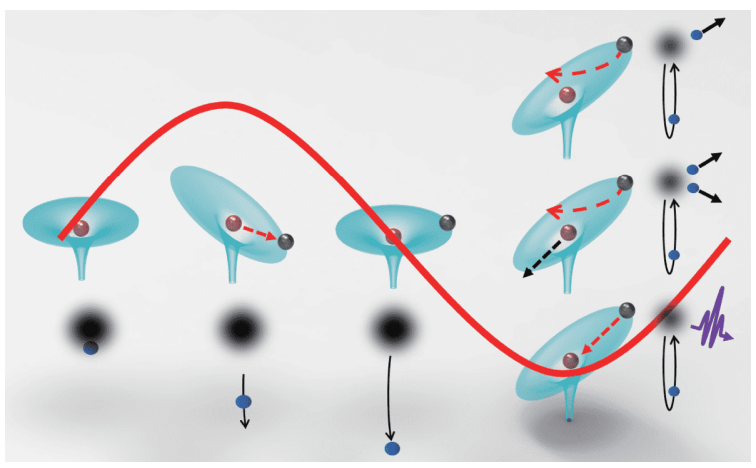

图 2 高次谐波产生的三步模型示意图. 电子被光电场(红色曲线)电 离, 并在光电场内运动; 当电场反向时, 电子加速飞回离子并与之相 互作用发生3种不同的物理过程. 右侧小图从上到下: 弹性碰撞, 电子 与离子中电子发生弹性散射, 能量不变, 观测结果是阈上电离; 非弹 性碰撞, 电子与离子中电子发生非弹性散射, 部分能量传递给另一个 电子, 观测结果是双电子电离; 电子与离子复合回到基态, 能量以一 个高能光子的形式释放, 观测结果是高次谐波产生. 势阱中棕红色小 球代表束缚态电子，黑色小球代表正在被电离或已经电离至连续态 的电子, 与黑色带箭头曲线一起的蓝色小球代表电子运动轨迹

Figure 2 Three-step model for HHG. The electron is ionized by the laser (red curve) and moves in the field. When the electric field is reversed, the electron accelerates back to the ion and may undergo one of the three different physical processes. Insets from top to bottom on the right: Elastic collision, the electron is scattered by the electrons of the ion elastically, its energy does not change, and ATI is observed; inelastic collision, the electron is scattered by an electron in the ion inelastically, the energy is transferred partially, double-ionization is observed; the electron recombines with the ion and returns to the ground state, its energy is released by a high-energy photon, HHG is observed. The brown-red balls in the potential well represent bound electrons, the black balls represent electrons that are being ionized or have been ionized into the continuous state, and the blue balls along with the black arrow curve represent the electron trajectories

谱大致上可分为 3 个部分：第一部分为微扰区，区内高 次谐波的级次较低，谐波效率随着级次的提升而快速 下降；第二部分为平台区，位于谐波光谱的中部，各级 次强度相差不大; 第三部分为截止区, 该区域的谐波级 次较高, 效率也随着级次的提升而下降, 也有可能是没 有干涉的连续光谱.

\section{2 阿秒脉冲的产生和孤立脉冲选通技术}

如前所述, $\mathrm{HHG}$ 在驱动飞秒激光(有时也称为基频 光)的每半个光周期产生一次，辐射出的极紫外脉冲宽 度小于半个光周期, 达到亚飞秒即阿秒量级 ${ }^{[16]}$. 频域中 梳齿状的HHG谱在时域上对应一个阿秒脉冲序列，或 称阿秒脉冲串. 利用飞秒脉冲作为驱动源, 并采用相应 的选通 (gating)方法，就可以从HHG的阿秒脉冲序列中 选出一个脉冲, 称为孤立阿秒脉冲, 如图3所示; 其与驱 动脉冲重复频率相同, 且精确同步 ${ }^{[17,20,37 \sim 39]}$. 例如, 振幅 

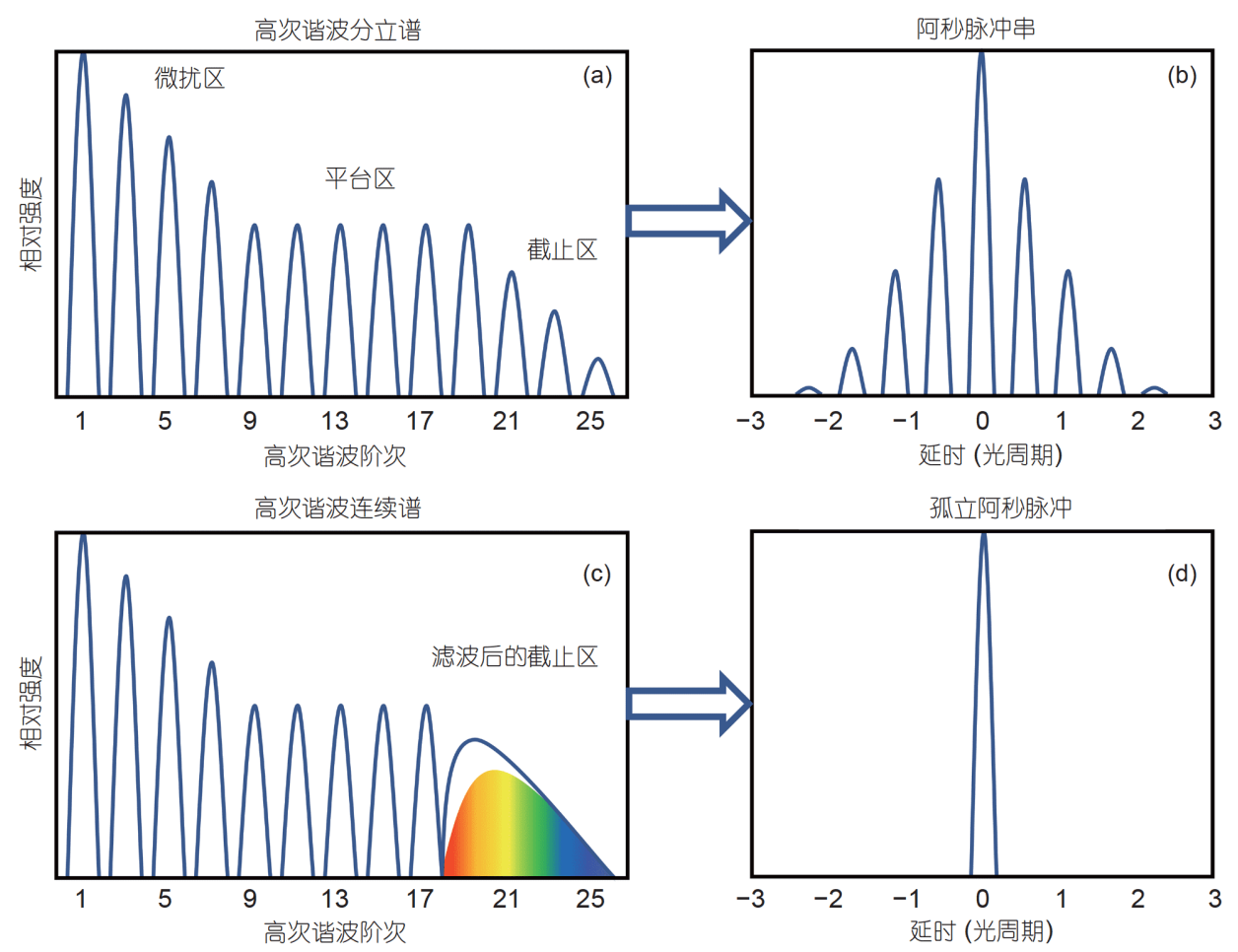

图 3 (网络版彩色)高次谐波及对应的阿秒脉冲特性. 高次谐波分立谱包括微扰区、平台区和截止区; 在时域上对应一个阿秒脉冲串. 在高次 谐波的截止区产生一段连续光谱, 将其滤波后在时域上对应一个孤立阿秒脉冲

Figure 3 (Color online) HHG and corresponding attosecond pulses. A high-order harmonic spectrum contains the perturbation region, the plateau region, and the cutoff region. A discrete high-order harmonic spectrum corresponds to a series of attosecond pulses in the time domain. A continuous spectrum in the cutoff region corresponds to an isolated attosecond pulse after being filtered out

选通 ${ }^{[23,26,28,30,40]}$ 就是利用载波包络相位(carrier-envelope phase, CEP)锁定 ${ }^{[41 ~ 48]}$ 的近单周期飞秒脉冲驱动产生

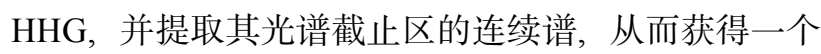
孤立阿秒脉冲. 使用负色散的金属膜(如铝、锆膜等)和 气体能够补偿脉冲的正色散, 进一步压缩阿秒脉冲, 甚 至可以直接使用单周期飞秒余弦脉冲，这样产生的 $\mathrm{HHG}$ 光谱只包含一个连续谱, 直接就是一个孤立阿秒 脉冲.

迄今人们已经发展了多种由气体HHG产生孤立阿

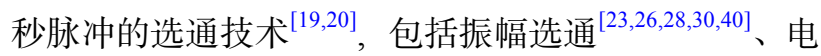
离选通 ${ }^{[49 \sim 54]}$ 、偏振选通 ${ }^{[17,25,29,55 ~ 57]}$ 、双光选 通 ${ }^{[27,58 \sim 61]}$ 、空间选通 ${ }^{[62 \sim 66]}$ 、干涉偏振选通 ${ }^{[67,68]}$ 及偏振 辅助的振幅选通 ${ }^{[69]}$ 等, 此外, 还有利用相位匹配的时域 窗口产生孤立阿秒脉冲的方案 ${ }^{[70]}$. 在这些选通技术中, 往往涉及的一个重要问题是CEP的锁定. CEP 是指脉冲 包络与内部电场峰值间的相位差, 其值决定了激光脉 冲电场的绝对形状. 在激光脉冲的产生与传输过程中, 色散、非线性效应以及外部环境因素等都会使得其 $\mathrm{CEP}$ 发生漂移 ${ }^{[1148]}$. 对于长脉冲, 由于一个脉冲内电场
周期较多, 因此CEP值对脉冲形状影响不大; 而对于少 周期脉冲, $\mathrm{CEP}$ 值的变化将会显著改变脉冲电场的真实 形状. 因此, 在研究少周期脉冲与物质相互作用时, 尤 其对于基于强场电离的高次谐波和阿秒脉冲产生实验, $\mathrm{CEP}$ 的锁定至关重要 ${ }^{[37,38,71,72]}$. 当电场峰值锁定在包络 峰值附近时, 才有可能获得宽光谱的孤立阿秒脉冲.

\section{1 振幅选通}

由三步模型可知，光电场中每半个周期产生一个 极紫外高次谐波脉冲, 如果需要得到单个阿秒脉冲, 就 需要尽可能短的飞秒脉冲. 理论上, 使用半周期的飞秒 脉冲就能直接产生孤立阿秒脉冲, 但是这样的光源很 难实现. 实验中, 我们往往只能够得到 1 2个周期左右 的飞秒脉冲 ${ }^{[6,73,74]}$. 使用多周期脉冲作为驱动源, 只能 够得到阿秒脉冲串.

小于两个周期的脉冲, 当CEP锁定在 0 或 $\pi$ 时, 脉冲 包络与电场的峰值位置重合，此时这个周期的电场最 强, 电离电子获得的动能最多, 辐射出的光子能量最高 (图4). 因此，在高次谐波光谱的截止区产生一段连续 


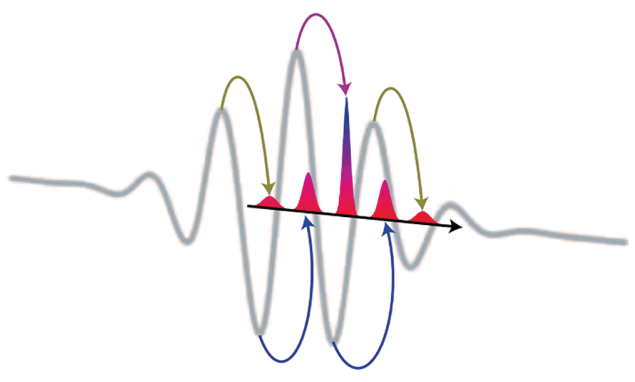

图 4 振幅选通原理. 带箭头的曲线标示出驱动飞秒脉冲中不同半 周期所产生的阿秒光谱. 修改自文献[20]中的图1

Figure 4 Amplitude gating. The arrow curves indicate the contributions of different half cycles to the total attosecond spectrum. Adapted from Fig. 1 in Ref. [20]

谱 $^{[20,23,26,28,72]}$ (图5), 该连续谱只来源于一个阿秒脉冲的 贡献，通过特定镀膜的带通反射镜或金属薄膜滤波片 分离出这段连续谱并补偿色散，就能够得到孤立阿秒 脉冲, 此为振幅选通(amplitude gating) ${ }^{[23,26,28,30,40]} .2013$ 年，中国科学院物理研究所利用钛宝石飞秒激光作为 驱动光源结合该技术, 得到了 160 as的结果 ${ }^{[28]} .2017$ 年, Gaumnitz等人 ${ }^{[30]}$ 采用中心波长 $1.8 \mu \mathrm{m}$ 、脉宽 $11 \mathrm{fs}$ (近两 个周期)的中红外飞秒激光作为驱动源与振幅选通技 术结合, 得到了脉冲宽度 43 as的孤立脉冲, 这也是目前 的最短阿秒脉冲世界纪录.

\section{2 电离选通}

电离选通(ionization gating) ${ }^{[49 \sim 54]}$ 与振幅选通一样, 选取CEP锁定飞秒脉冲驱动产生HHG的截止区连续谱 进行滤波, 分离孤立阿秒脉冲. 电离选通依赖于驱动脉 冲前沿, 在产生高次谐波的同时, 在气体靶内产生大量 电离，以致后续电场到达时反应区内等离子体密度过 高，即此时自由电子引起的负色散不能被剩余中性气 体原子的正色散补偿，无法满足HHG辐射的相位匹配 条件, 因此其后的谐波辐射无法发生. 该条件下产生的 $\mathrm{HHG}$ 只对应于驱动脉冲前沿所产生的少数几个阿秒脉 冲, 再与振幅选通类似采用带通滤波, 选择截止区的连 续谱就可以得到孤立阿秒脉冲。该方法还可以通过改 变驱动脉冲的光强，调节所得阿秒脉冲的光谱中心位 置, 实现输出调谐 ${ }^{[52]}$.

\section{3 偏振选通}

振幅选通和电离选通都是利用截止区的连续谱分 离孤立阿秒脉冲. 如果利用亚周期的线偏振余弦脉冲 光场，就能直接得到对应一个孤立阿秒脉冲的超连续

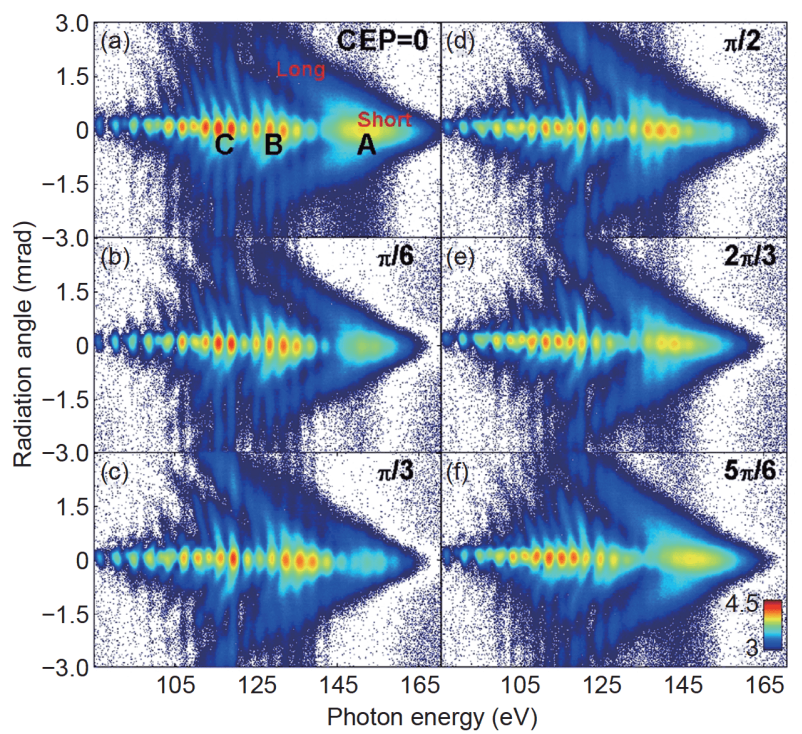

图 5 实验中不同 $\mathrm{CEP}$ 下少周期脉冲产生的箭头状高次谐波光谱. (a) $\mathrm{CEP}=0$; (b) $\mathrm{CEP}=\pi / 6$; (c) $\mathrm{CEP}=\pi / 3$; (d) $\mathrm{CEP}=\pi / 2$; (e) $\mathrm{CEP}=2 \pi / 3$; (f) $\mathrm{CEP}=5 \pi / 6$. 修改自文献[72]中的图 1

Figure 5 Experimental arrow-like high-harmonic spectra with different carrier envelope phases (CEP). (a) $\mathrm{CEP}=0$; (b) $\mathrm{CEP}=\pi / 6$; (c) $\mathrm{CEP}=\pi$ / 3; (d) $\mathrm{CEP}=\pi / 2$; (e) $\mathrm{CEP}=2 \pi / 3$; (f) $\mathrm{CEP}=5 \pi / 6$. Adapted from Fig. 1 in Ref. [72]

高次谐波谱, 其宽度可以支持极短的阿秒脉冲. 一般而 言，非线偏光很难产生高次谐波，因为电子在圆或椭圆 偏振的电场下，电场力并不指向原子的方向，电子很难 飞回初始位置与原子复合. 而偏振选通(polarization gating, PG $)^{[17,25,29,55 \sim 57]}$ 利用两束电场旋转方向相反的圆偏 光, 并设置特定相对延时, 使两个圆偏光的电场叠加后, 在叠加场的中心附近形成一处半个周期的线偏光场.

如图6所示，实验中将小于两个光学周期的线偏振 脉冲通过特定厚度的石英片，其光轴与光场偏振方向 成 $45^{\circ}$, 分解成具有特定延时且互相垂直的两线偏脉冲; 再经过一个 $1 / 4$ 波片，将这两个脉冲变成两个反向旋转 的圆偏振脉冲, 并在此特定延时下, 在两脉冲重合的中 心形成一处接近半周期的线偏振光场 ${ }^{[25,75]}$. 由于其前 后光场都为圆偏光，因此高次谐波产生过程中，只能在 此附近出现由三步过程产生的辐射，得到一个宽带的 连续高次谐波光谱，对应一个孤立阿秒脉冲. 2017年， $\mathrm{Li}$ 等人 ${ }^{[29]}$ 利用偏振选通获得了中心波长 $170 \mathrm{eV} 、 53$ as 的孤立脉冲, 但是由于金属膜补偿带宽的限制, 并没有 压缩到其带宽支持的 20 as.

\section{4 双光选通}

双光选通(double optical gating, DOG) $)^{[27,32 \sim 34,58 \sim 61]}$ 


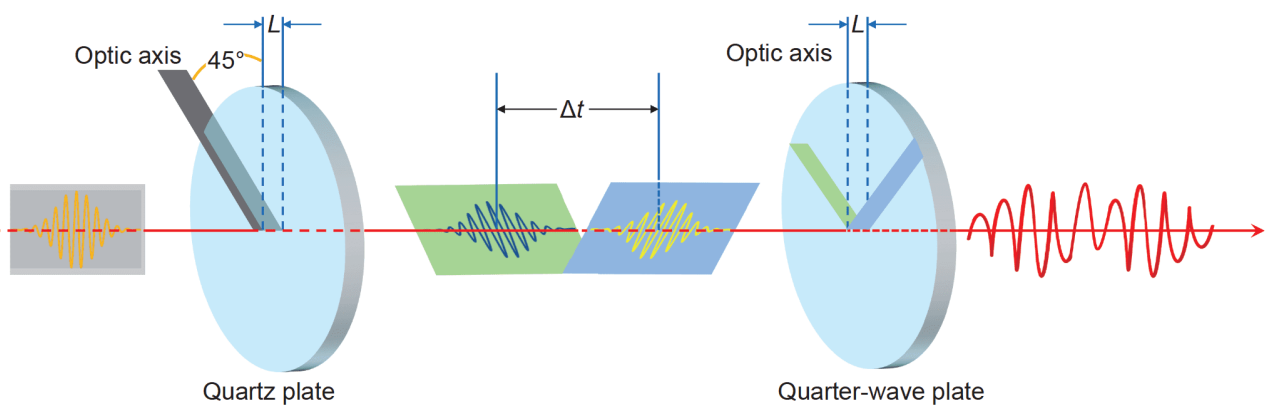

图 6 偏振度随时间变化脉冲的产生原理示意图

Figure 6 The principle for producing a pulse with a time-dependent ellipticity

是双色场和偏振选通的组合. 通过对飞秒激光倍频引 人二次谐波场(强度约为基频光的 10\% 20\%)并与基频 脉冲叠加, 选取合适的叠加相位, 使原本正负电场强度 和形状对称的基频光场变为不对称的双色场(图7). 相 比于单色基频场, 此双色场正向电场得到加强, 负电场 被削弱以致无法支持HHG辐射，使HHG过程中原本每 半周期产生一个阿秒脉冲变成每周期才产生一个 ${ }^{[76]}$ (图7). 这样, 对选通门宽的要求就从半个光周期降低至 一个周期，从而可以使用约 $12 \mathrm{fs}$ 的激光脉冲(中心波长 在800 nm附近)构成选通以产生孤立阿秒脉冲，即偏振 选通需要小于两个周期的光脉冲，加人倍频场后则可 以使用小于 4 个周期的光脉冲. 2009 年, Mashiko等人 ${ }^{[77]}$ 利用双光选通获得了光子能量覆盖28 620 eV(波长 2 45 nm)的XUV超连续光谱, 其变换极限达到 16 as. 2012 年, Zhao等人 ${ }^{[27]}$ 使用双光选通得到了 67 as的孤立 脉冲. 2020 年, 国内 3 个团队的孤立阿秒脉冲产生实验 也都采用了双光选通方案 ${ }^{[32 \sim 34]}$.

双光选通建立在偏振选通的基础上，对基频光的 脉宽要求放宽到小于 12 fs. 而广义双光选通 (generalized double optical gating, GDOG) ${ }^{[60,78,79]}$ 将对基频光的脉宽 要求进一步放宽到28 30 fs. 图8所示是GDOG的原理示 意图. 由于使用长脉冲的一个致命问题是反应区内的 原子在合成的线偏振场到达之前就全部被电离了，使 得线偏振场无法产生阿秒脉冲, 因此, 相比DOG, 在光 路中增加一布鲁斯特窗片, 将DOG光路中的圆偏振变 成椭圆偏振. 此光路通过对长脉冲增加相应的延时, 得 到与DOG相同的选通宽度; 并通过降低产生场方向的 整体电场强度, 解决了基态原子过度电离的问题.

\section{5 空间选通}

目前最为典型的空间选通技术是所谓的阿秒灯塔

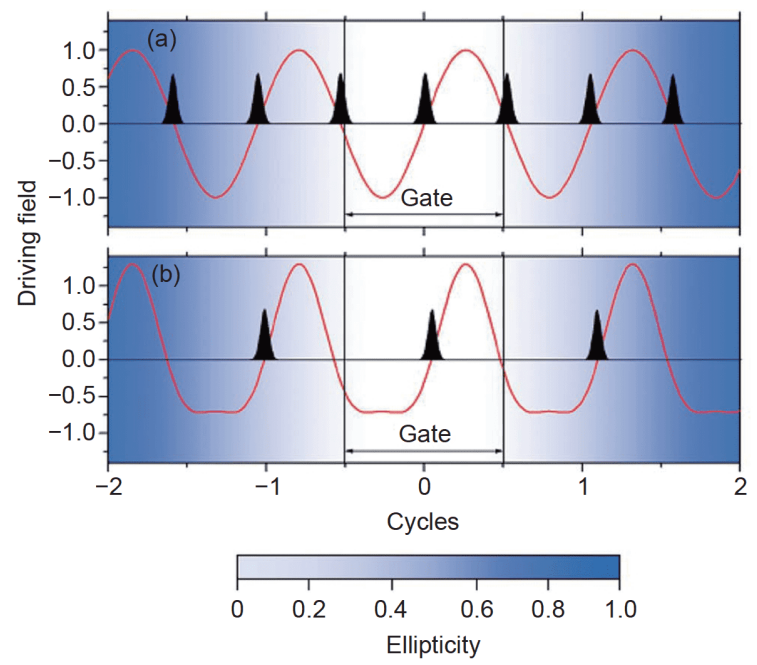

图 7 对应单色场(a)和双色场(b)的偏振选通的驱动光场成分 ${ }^{[59]}$. 红 实线表示驱动光场. 两条坚线表示选通门宽. 填充的曲线表示只有 驱动场时辐射出的阿秒脉冲. 背景颜色的深浅代表偏振选通的强弱.

Copyright (2021) by the American Physical Society

Figure 7 The driving field components for PG correspond to without (a) and with (b) the second harmonic field, respectively ${ }^{[5]}$. The driving field is shown as the red line. The two vertical lines represent the gate width. Here, the filled curves are the attosecond pulses emitted when the driving fields alone are applied. The background color shows the strength of the PG. Copyright (2021) by the American Physical Society

(attosecond lighthouse $)^{[62,63,80]}$ 方法，该方法依赖于驱动 光的波前倾斜，以产生传播方向空间分离的孤立阿秒 脉冲. 当由波前倾斜的飞秒激光驱动 $\mathrm{HHG}$ 产生阿秒脉 冲时，每半个光周期产生的阿秒脉冲将沿不同的方向 发射，每个脉冲都垂直于其产生时驱动激光的瞬时波 前, 且具有比驱动激光束小得多的发散角. 因此, 如果 在驱动激光的半个周期内波前旋转大于阿秒脉冲的发 散角, 则阿秒灯塔效应可以通过远场的空间滤波器选 出孤立阿秒脉冲. 2013年, Kim等人 ${ }^{[63]}$ 在理论和实验上 讨论了利用阿秒灯塔能够产生孤立阿秒脉冲. 实验中 


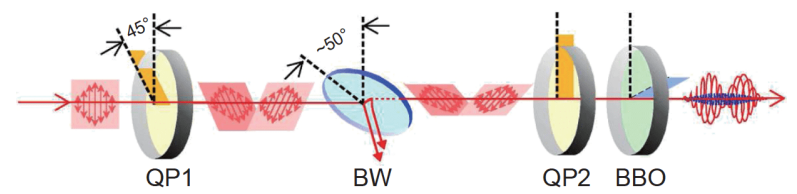

图 $8 \mathrm{GDOG}$ 光学元件包括石英波片 $(\mathrm{QP} 1)$ 、布鲁斯特窗 $(\mathrm{BW}) 、$ 第 二个石英波片( $\mathrm{QP} 2)$ 和 $\mathrm{BBO}$ (beta barium borate, 偏嗍酸钡)倍频晶体. 修改自文献[60]中的图2

Figure 8 GDOG optics consist of a quartz plate (QP1), a Brewster window $(\mathrm{BW})$, a second quartz plate $(\mathrm{QP} 2)$, and a $\mathrm{BBO}$ crystal. Adapted from Fig. 2 in Ref. [60]

每个半周期的电场都存在一个固定的角度差，使得其 产生的阿秒脉冲辐射方向都不相同，最终可以在空间 分辨开来. 2016年，Hammond等人 ${ }^{[80]}$ 用远场光阑截取 阿秒灯塔效应产生的空间分离的 3 个阿秒脉冲，并用阿 秒条纹相机分别测量了脉宽，确认了阿秒灯塔方法的 有效性.

非共线光学选通 ${ }^{[6466]}$ 是另一种空间选通方式，两 束CEP锁定的飞秒脉冲以非共线的方式聚焦在气体靶 上，并在气体靶位置时空重合. 通过延时的调节，两个 电场产生调制，会产生类似于阿秒灯塔的电场波前形 变. 每个半周期的电场传播方向会产生一个固定的角
度差，其产生的高次谐波辐射都会沿着这个固定角度 差逐渐分开——垂直于其产生时驱动激光的瞬时波前, 同样能够在空间上选出孤立阿秒脉冲(图9).

\section{6 干涉偏振选通}

干涉偏振选通(interferometric polarization gating) 可以通过多周期脉冲产生单个阿秒辐射 ${ }^{[67,68]}$ ，其原理 是基于偏振选通将光束分为两臂，每一臂都有干涉及 延时控制装置. 其中一臂调节延时, 使干涉脉冲变成类 似超高斯的形状; 另一臂则将两束脉冲干涉形成中心 凹陷脉冲, 再通过半波片将线偏振光转换成中心凹陷 的圆偏振光. 最终将两臂光束合成，凹陷处仍为线偏 光，而其他部分的线偏光则与圆偏光合成为椭圆偏光. 如果各部分相位延时控制得足够精密，理论上凹陷处 会存在一个周期左右的线偏光用来产生单个阿秒脉冲. 这种方法虽然理论上能够用长周期的脉冲最终产生单 个阿秒脉冲，但是其光路的复杂程度和各臂控制精度 的要求使其实验具有极大的挑战性.

\section{7 偏振辅助的振幅选通}

2016年, Timmers等人 ${ }^{[69]}$ 提出了基于振幅选通技术

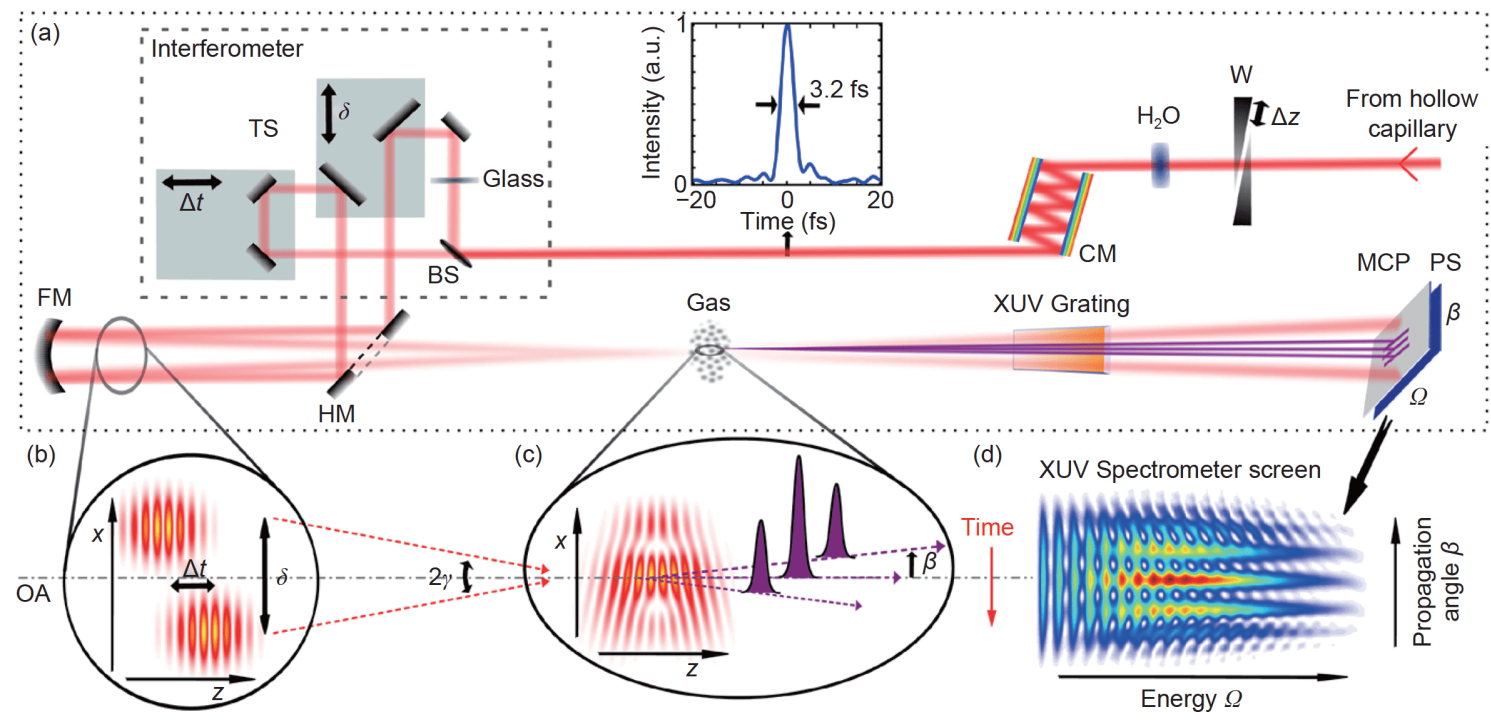

图 9 (a) 非共线选通实验装置示意图. W, 一对电动尖䢃; $\mathrm{H}_{2} \mathrm{O}$, 水槽; $\mathrm{CM}$, 啁啾镜; $\mathrm{BS}$, 分束器; TS, 平移台; $\mathrm{HM}$, 带孔镜; FM, 聚焦镜 $(f=$ $400 \mathrm{~mm}$ ); Gas, 氩气; MCP, 多通道板探测器; PS, 苂光屏; OA, 光轴. (b) 聚焦前, 在远场的两路红外激光脉冲 $(\Delta t>0)$ 和(c)在气体靶处的电场叠加 形成选通条件, 以及(d)角分离的 XUV连续光谱(模拟结果)显示了时间到角度的映射. Reprinted Fig. 1 with permission from Ref. [65] (C) The Optical Society

Figure 9 (a) Schematic of the experimental setup. W, motorized pair of wedges; $\mathrm{H}_{2} \mathrm{O}$, water cell; CM, chirped mirrors; BS, beam splitter; TS, translation stages; HM, holey mirror; FM, focusing mirror $(f=400 \mathrm{~mm})$; Gas, argon; MCP, multichannel plate; PS, phosphor screen; OA, optical axis. (b) Illustration of the IR laser pulses in the far-field before focusing $(\Delta t>0)$ and (c) at the position of the gas cell in gating conditions, and (d) of the angularly separated spectral XUV continua (simulation) showing the time-to-angle mapping. Reprinted Fig. 1 with permission from Ref. [65] (C) The Optical Society 
并使用偏振变化进行辅助的单阿秒产生技术PASSAGE(polarization assisted amplitude gate). 此方案采用 与偏振选通类似的光路, 但是提供延时的石英片的厚 度只有偏振选通中使用的一半, 提供的延时也只有一 半. 因此, 与偏振选通叠加场中心附近产生半个周期的 线偏振场不同, PASSAGE的叠加场中线偏振场部分可 长至一个光周期左右. 其结果就是在叠加场中心附近 有不止一个能够产生高次谐波的半周期，与振幅选通 方案中使用的近单周期驱动脉冲电场类似; 但是, 由于 左右旋偏振场之间延时较小, 线偏振部分的电场强度 较高, 所产生的高次谐波光子能量也较高. 与振幅选通 类似, 再利用金属膜滤出截止区的连续谱, 即可获得孤 立阿秒脉冲. 与振幅选通相比, 此方案适度放宽了 对驱动激光脉宽的要求; 与偏振选通相比, 此方案的产 生电场强度更高, 所获得孤立阿秒脉冲的光子能量也 更高.

\section{8 相位匹配的时域窗口产生孤立阿秒脉冲}

2014年，Chen等人 ${ }^{[70]}$ 提出利用相位匹配的时域窗 口产生孤立阿秒脉冲. HHG过程中相位匹配直接影响 能量、光谱、脉宽等重要参数. 他们首次提出, 多周期 驱动脉冲与气体相互作用时, 在峰值附近存在一个相 位匹配较好的窗口, 可用于产生孤立阿秒脉冲. 在驱动 脉冲前沿, 由于气体靶中主要还是中性原子, 激光相速 度小于光速而产生相位失配; 脉冲后沿随着原子被电 离, 等离子体密度升高导致激光相速度大于光速也产 生相位失配. 最终, 只在脉冲的中间形成一个相位匹配 窗口, 此处的电场能够产生稳定的相位匹配的高次谐 波; 如能将窗口控制在半个光周期内, 即可产生孤立阿 秒脉冲. 但是, 此方法对焦点处激光参数的控制有较为 苛刻的要求.

该实验组搭建了一台高精度阿秒干涉自相关仪(分 辨率高达 $1.5 \mathrm{as}$ ), 用于测量在改变不同波长驱动光的峰 值功率密度时产生的单个阿秒脉冲和脉冲串的宽度. 实验发现, 长波长脉冲在一定峰值功率密度下能够产 生单个阿秒脉冲辐射. 实验使用了驱动光参数分别为 $0.8 \mu \mathrm{m} 、 24 \mathrm{fs}, 1.3 \mu \mathrm{m} 、 35 \mathrm{fs}$ 和 $2.0 \mu \mathrm{m} 、 90 \mathrm{fs}$ 的3种光 源; 在改变峰值功率密度进而改变相位匹配窗口宽度 的过程中, 发现 $2 \mu \mathrm{m}$ 的驱动光产生了单个阿秒脉冲. 他 们也数值模拟了 $2 \mu \mathrm{m}$ 波长下气压与相位匹配窗口的关 系, 计算与实验结果符合, 从理论上验证了该方法的可 行性.

\section{3 强场等离子体高次谐波产生阿秒脉冲}

超强激光聚焦在固体靶表面也能够产生HHG，对 此过程的解释主要有 3 种机制: 相干尾波辐射 ${ }^{[81]}$ 、振荡 反射镜 ${ }^{[82]}$ 以及相干同步辐射 ${ }^{[83 ~ 85]}$, 原理都是通过强光 场(超过 $10^{16} \mathrm{~W} / \mathrm{cm}^{2}$ ) 在靶上产生等离子体, 等离子体内 的电子与激光场相互作用产生高速运动释放出光子. 如振荡反射镜, 其机制是等离子体中电子密度高的部 分反射人射光, 电子和真空的界面受到外场驱动在垂 直界面方向振荡, 相当于一个振荡的反射镜; 由于多普 勒效应, 反射光中会出现不同的频率成分, 如果反射镜 的振荡频率与人射光频相同, 反射方向会出现各阶次 的谐波. 等离子体高次谐波能量比经典三步模型产生 的气体高次谐波高出数个数量级. 2019年, Wang等 人 $^{[86]}$ 首次提出, 可以利用振荡反射镜产生带轨道角动 量的阿秒脉冲 ${ }^{[87]}$.

2020 年, Zhang等人 ${ }^{[88]}$ 提出了双色场利用相干同 步辐射产生孤立阿秒脉冲的理论模型. 他们提到, 单色 场下，相干同步辐射每一个光周期会产生两次阿秒辐 射, 同时有电子溢出, 导致薄膜靶表面附近的电子密度 降低, 不利于后续辐射的产生. 利用双色场改变光场振 荡, 使其每周期只保留一个完好的电场, 而在另一个强 度降低了的电场中阿秒辐射较少, 电子溢出也较少, 这 就维持了一个较高的电子密度; 当激光峰值到达时, 薄 膜表面附近的电子会形成一个强束共同加速, 并通过 相干同步辐射产生一个强度远高于其他周期的孤立阿 秒脉冲(图10). 之后, 他们的进一步计算又预测, 使用双 层膜靶可以产生更高强度的孤立阿秒脉冲 ${ }^{[89]}$.

\section{4 阿秒脉冲的色散和压缩}

飞秒脉冲的色散来源于传播过程中不同波长成分 在材料中的相速度不同, 而阿秒脉冲产生时存在色 散 ${ }^{[16,90]}$. 在飞秒驱动脉冲的一个振荡周期内(图11中虚 线表示), 不同时刻电离出的电子在驱动光场中飞行的 距离不同(图11中彩色线), 往返一次回到离子附近并与 其复合的时刻不同, 所携带的动能不同, 进而辐射出的 光子能量也不同. 而这些光子构成一个阿秒脉冲, 并最 终表现为同一个阿秒脉冲内不同能量光子的初始相位 不同, 因此阿秒脉冲在形成时就有色散, 称为本征色 散 ${ }^{[91]}$.

对飞秒激光脉冲进行压缩时往往使用啁啾镜、色 散压缩(Gires-Tournois interferometer, GTI)镜、光栅或 

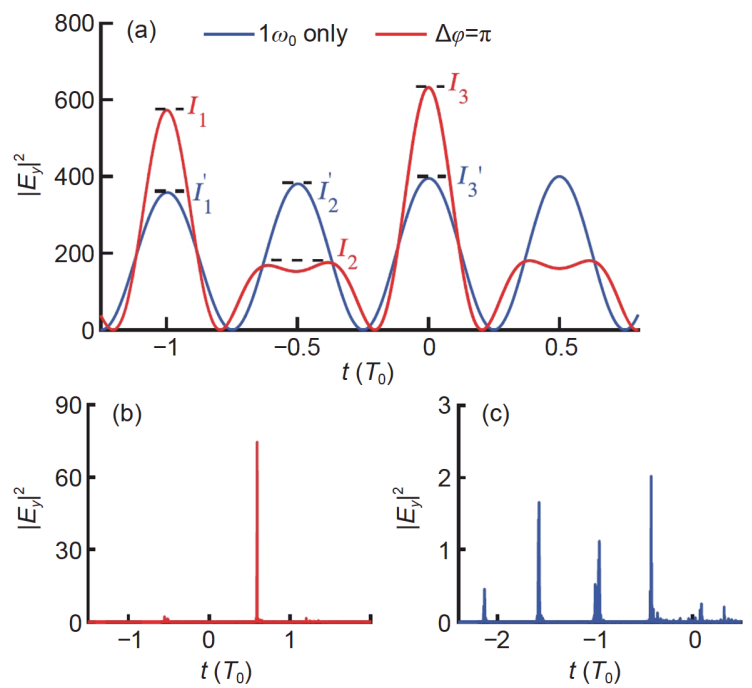

图 10 一维模型计算结果显示单色场和双色场的显著区别 ${ }^{[88]}$. (a) 单色场与双色场的光强变化, 红色为双色场, 蓝色为单色场. 反射出 的XUV辐射在双色场(b)下产生一个极强的脉冲, 高于单色场(c)下产 生的多个脉冲近40倍. Copyright (2021) by the American Physical Society

Figure 10 1D simulation highlighting the difference between twocolor with one-color interactions ${ }^{[88]}$. (a) The temporal intensity variation (red line for two color, blue line for one color). The reflected XUV emission in (b) and (c) shows a single attosecond burst for the two-color case which is about $\times 40$ brighter than the one-color pulse train. Reprinted Fig. 1 with permission from Ref. [88]. Copyright (2021) by the American Physical Society

棱镜对进行色散补偿，但是极紫外波段的类似光学元 件效率极低且制作困难，因此阿秒色散目前主要使用 材料来进行补偿, 通常使用金属薄膜或某些气体. 这些 材料在XUV至X射线波段通常会有一个透射窗口，在 这个窗口的低能量光谱部分, 材料具有负色散, 可用于 对拥有正本征色散的阿秒脉冲进行色散补偿和压 缩 ${ }^{[92]}$. 图12展示了几种材料的透过率和色散曲线.

2019年, Chang ${ }^{[93]}$ 提出了利用气体补偿极紫外阿秒 脉冲色散的想法, 并计算模拟了这种方法的可行性, 结 果显示, 在高于 $500 \mathrm{eV}$ 的情况下, 气体不仅具有较高的 透过率, 还能很好地补偿色散.

\section{5 总结与展望}

高次谐波的出现使得超快光学从飞秒领域跨进了 阿秒世界的大门. 相关的诸多技术如CEP锁定技术、超 连续展宽技术、电子飞行时间测量技术等，带动着超 快光学及其应用多方面的发展. 阿秒选通技术的发展 不仅为得到更短脉宽、更高能量的阿秒脉冲提供了帮 助, 也放宽了对基频光脉宽的要求, 使人们能够更便捷

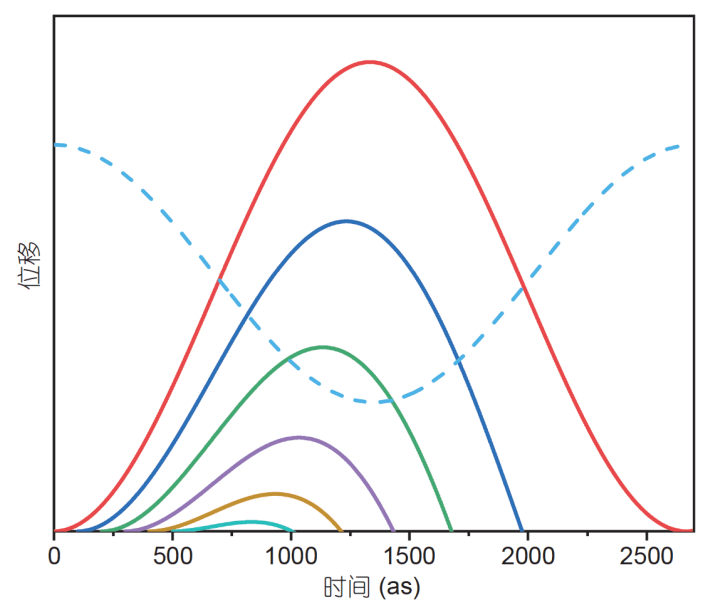

图 11 阿秒脉冲的本征色散产生原理. 电子在光场内不同时刻被电 离, 在光电场作用下与离子复合的时间也不相同, 导致辐射出的不同 能量的光子存在延迟

Figure 11 Intrinsic chirp of attosecond pulse generation. Electrons, ionized at different times in the driving laser field, recombine with the ions under the action of the laser field at different times. Therefore, XUV photons of different energies emit at different times

地得到并使用阿秒脉冲, 并实现对阿秒脉冲的操控 ${ }^{[94]}$.

阿秒脉冲是目前人类能够掌握的时间尺度最短的 工具. 利用阿秒相干脉冲, 能够开展对电子动力学超快 时间分辨的研究，使人们对物质结构的研究从原子分 子推进到原子内部电子的运动，最终能够实现对原子 内电子动力学过程的控制. 利用阿秒脉冲, 我们能够跟 踪化学反应中的电子，了解化学反应的进程; 也可以在

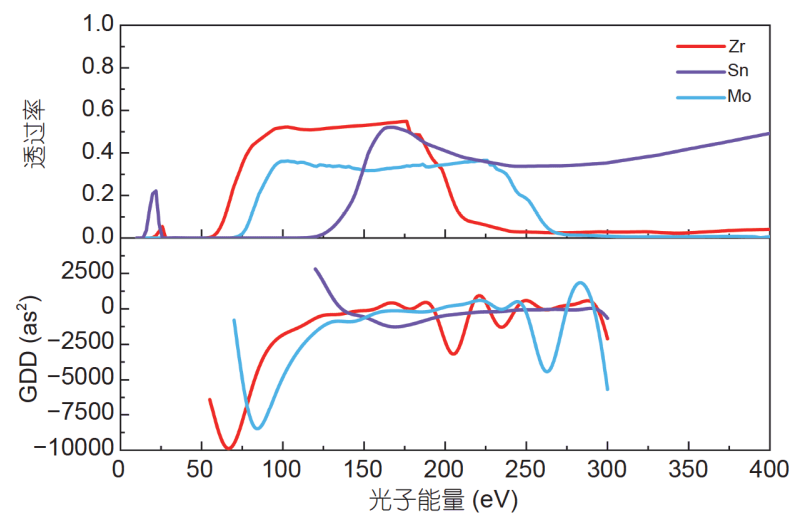

图 12 不同材料的透过率及色散. 上图为透过率，下图为群延迟色 散(GDD). 红色线代表锆膜(Zr), 紫色线代表锡膜(Sn), 蓝色线代表锄 膜(Mo)

Figure 12 Transmittance and dispersion of different materials. The upper figure shows the transmittance, and the lower figure shows the group delay dispersion (GDD). The red lines represent the zirconium film ( $\mathrm{Zr})$, the purple lines represent the tin film (Sn), and the blue lines represent the molybdenum film (Mo) 
半导体内实现绝缘体与半导体的光致转变, 实现新的 光开关, 突破目前半导体电子器件开关速度的瓶颈. 并 且, 随着各种孤立阿秒脉冲产生技术的成熟, 实验室中 桌面级的高次谐波光源产生的光谱能够达到几百电子 伏特, 甚至覆盖水窗波段, 这样的脉冲非常适合用于生
物医学的研究, 是活体生物样本X射线显微成像的最佳 光谱范围. 阿秒技术有望揭示光伏效应的超快动力学 过程, 并对该过程进行操控, 提升光伏材料能量转换效 率. 阿秒脉冲技术的进步也可能是实现入秒脉冲并让 研究进人原子核内部的关键.

\section{参考文献}

1 Maiman T H. Stimulated optical radiation in ruby. Nature, 1960, 187: 493-494

2 Liu H, Wang G, Jiang J, et al. Sub-10-fs pulse generation from a blue laser-diode-pumped Ti:sapphire oscillator. Chin Opt Lett, 2020, 18: 071402

3 Sutter D H, Steinmeyer G, Gallmann L, et al. Semiconductor saturable-absorber mirror-assisted Kerr-lens mode-locked Ti:sapphire laser producing pulses in the two-cycle regime. Opt Lett, 1999, 24: 631-633

4 He P, Liu Y, Zhao K, et al. High-efficiency supercontinuum generation in solid thin plates at 0.1 TW level. Opt Lett, 2017, 42: 474

5 Huang P, Fang S, Gao Y, et al. Simple method for simultaneous long-term stabilization of relative timing and carrier-envelope phase in waveform synthesis. Appl Phys Lett, 2019, 115: 031102

6 Silva F, Alonso B, Holgado W, et al. Strategies for achieving intense single-cycle pulses with in-line post-compression setups. Opt Lett, 2018, 43: 337

7 Hassan M T, Luu T T, Moulet A, et al. Optical attosecond pulses and tracking the nonlinear response of bound electrons. Nature, 2016, 530: 66-70

8 Damon E K, Tomlinson R G. Observation of ionization of gases by a ruby laser. Appl Opt, 1963, 2: 546

9 Meyerand R G, Haught A F. Gas breakdown at optical frequencies. Phys Rev Lett, 1963, 11: 401-403

10 Keldysh L V. Ionization in the field of a strong electromagnetic wave. Zh Eksp Teor Fiz, 1964, 47: 1945 [Sov Phys JETP, 1965, 20: 1307]

11 Agostini P, Fabre F, Mainfray G, et al. Free-free transitions following six-photon ionization of xenon atoms. Phys Rev Lett, 1979, 42: 1127-1130

12 McPherson A, Gibson G, Jara H, et al. Studies of multiphoton production of vacuum-ultraviolet radiation in the rare gases. J Opt Soc Am B, 1987, 4: $595-601$

13 Ferray M, L'Huillier A, Li X F, et al. Multiple-harmonic conversion of 1064 nm radiation in rare gases. J Phys B-At Mol Opt Phys, 1988, 21: L31L35

14 Kuchiev M Yu. Atomic antenna. Pis'ma Zh Eksp Teor Fiz, 1987, 45: 319 [JETP Lett, 1987, 45: 404]

15 Schafer K J, Yang B, Dimauro L F, et al. Above threshold ionization beyond the high harmonic cutoff. Phys Rev Lett, 1993, 70: 1599-1602

16 Corkum P B. Plasma perspective on strong field multiphoton ionization. Phys Rev Lett, 1993, 71: 1994-1997

17 Corkum P B, Burnett N H, Ivanov M Y. Subfemtosecond pulses. Opt Lett, 1994, 19: 1870-1872

18 Lewenstein M, Balcou P, Ivanov M Y, et al. Theory of high-harmonic generation by low-frequency laser fields. Phys Rev A, 1994, 49: 2117-2132

19 Calegari F, Sansone G, Stagira S, et al. Advances in attosecond science. J Phys B-At Mol Opt Phys, 2016, 49: 062001

20 Chini M, Zhao K, Chang Z. The generation, characterization and applications of broadband isolated attosecond pulses. Nat Photonics, 2014, 8: $178-186$

21 Paul P M, Toma E S, Mullot G, et al. Observation of a train of attosecond pulses from high harmonic generation. Science, 2001, 292: 1689-1692

22 Hentschel M, Kienberger R, Spielmann C, et al. Attosecond metrology. Nature, 2001, 414: 509

23 Kienberger R, Goulielmakis E, Uiberacker M, et al. Atomic transient recorder. Nature, 2004, 427: 817-821

24 Itatani J, Quéré F, Yudin G L, et al. Attosecond streak camera. Phys Rev Lett, 2002, 88: 173903

25 Sansone G, Benedetti E, Calegari F, et al. Isolated single-cycle attosecond pulses. Science, 2006, 314: 443-446

26 Goulielmakis E, Schultze M, Hofstetter M, et al. Single-cycle nonlinear optics. Science, 2008, 320: 1614-1617

27 Zhao K, Zhang Q, Chini M, et al. Tailoring a 67 attosecond pulse through advantageous phase-mismatch. Opt Lett, 2012, $37: 3891$

28 Zhan M J, Ye P, Teng H, et al. Generation and measurement of isolated 160-attosecond XUV laser pulses at $82 \mathrm{eV}$. Chin Phys Lett, 2013 , 30: 093201

29 Li J, Ren X, Yin Y, et al. 53-attosecond X-ray pulses reach the carbon K-edge. Nat Commun, 2017, 8: 186

30 Gaumnitz T, Jain A, Pertot Y, et al. Streaking of 43-attosecond soft-X-ray pulses generated by a passively CEP-stable mid-infrared driver. Opt Express, 2017, 25: 27506-27518

31 Peng L Y, Jiang W C, Geng J W, et al. Tracing and controlling electronic dynamics in atoms and molecules by attosecond pulses. Phys Rep, 2015, 575: 1-71

32 Yang Z, Cao W, Chen X, et al. All-optical frequency-resolved optical gating for isolated attosecond pulse reconstruction. Opt Lett, 2020, 45: 567 
33 Wang X, Wang L, Xiao F, et al. Generation of 88 as isolated attosecond pulses with double optical gating. Chin Phys Lett, 2020, 37: 023201

34 Wang X L, Xu P, Li J, et al. Isolated attosecond pulse with 159 as duration measured by home built attosecond streaking camera (in Chinese). Chin J Laser, 2020, 47: 0415002 [王向林, 徐鹏, 李捷, 等. 利用自研阿秒条纹相机测得 159 as孤立阿秒脉冲. 中国激光, 2020, 47: 0415002]

35 Muller H G. Reconstruction of attosecond harmonic beating by interference of two-photon transitions. Appl Phys B, 2002, 74: S17-S21

36 Zhao K, Gao Y, Zhu X, et al. Principle and technology of attosecond pulse characterization (in Chinese). Chin Sci Bull, 2021, 66: 835-846 [赵昆, 高亦谈, 朱孝先, 等. 阿秒脉冲测量原理和技术研究进展. 科学通报, 2021, 66: 835-846]

37 Krausz F, Ivanov M. Attosecond physics. Rev Mod Phys, 2009, 81: 163-234

38 Chang Z, Corkum P. Attosecond photon sources: The first decade and beyond. J Opt Soc Am B, 2010, 27: B9

39 Sansone G, Poletto L, Nisoli M. High-energy attosecond light sources. Nat Photonics, 2011, 5: 655-663

40 Witting T, Frank F, Okell W A, et al. Sub-4-fs laser pulse characterization by spatially resolved spectral shearing interferometry and attosecond streaking. J Phys B-At Mol Opt Phys, 2012, 45: 074014

41 Xu L, Hänsch T W, Spielmann C, et al. Route to phase control of ultrashort light pulses. Opt Lett, 1996, 21: 2008-2010

42 Telle H R, Steinmeyer G, Dunlop A E, et al. Carrier-envelope offset phase control: A novel concept for absolute optical frequency measurement and ultrashort pulse generation. Appl Phys B, 1999, 69: 327-332

43 Jones D J, Diddams S A, Ranka J K, et al. Carrier-envelope phase control of femtosecond mode-locked lasers and direct optical frequency synthesis. Science, 2000, 288: 635-639

44 Apolonski A, Poppe A, Tempea G, et al. Controlling the phase evolution of few-cycle light pulses. Phys Rev Lett, 2000, 85: 740-743

45 Kakehata M, Takada H, Kobayashi Y, et al. Single-shot measurement of carrier-envelope phase changes by spectral interferometry. Opt Lett, 2001, 26: $1436-1438$

46 Paulus G G, Grasbon F, Walther H, et al. Absolute-phase phenomena in photoionization with few-cycle laser pulses. Nature, 2001, 414: 182-184

47 Fortier T M, Jones D J, Jun Ye D J, et al. Highly phase stable mode-locked lasers. IEEE J Sel Top Quantum Electron, 2003, 9: 1002-1010

48 Baltuska A, Uiberacker M, Goulielmakis E, et al. Phase-controlled amplification of few-cycle laser pulses. IEEE J Sel Top Quantum Electron, 2003, 9: 972-989

49 Cao W, Lu P, Lan P, et al. Single-attosecond pulse generation with an intense multicycle driving pulse. Phys Rev A, 2006, 74: 063821

50 Pfeifer T, Jullien A, Abel M J, et al. Generating coherent broadband continuum soft-X-ray radiation by attosecond ionization gating. Opt Express, 2007, 15: 17120

51 Jullien A, Pfeifer T, Abel M J, et al. Ionization phase-match gating for wavelength-tunable isolated attosecond pulse generation. Appl Phys B, 2008, 93: 433-442

52 Abel M J, Pfeifer T, Nagel P M, et al. Isolated attosecond pulses from ionization gating of high-harmonic emission. Chem Phys, 2009, 366: 9-14

53 Thomann I, Bahabad A, Liu X, et al. Characterizing isolated attosecond pulses from hollow-core waveguides using multi-cycle driving pulses. Opt Express, 2009, 17: 4611-4633

54 Ferrari F, Calegari F, Lucchini M, et al. High-energy isolated attosecond pulses generated by above-saturation few-cycle fields. Nat Photonics, 2010, 4: 875-879

55 Chang Z. Single attosecond pulse and XUV supercontinuum in the high-order harmonic plateau. Phys Rev A, 2004, 70: 043802

56 Chang Z. Chirp of the single attosecond pulse generated by a polarization gating. Phys Rev A, 2005, 71: 023813

57 Sola I J, Mével E, Elouga L, et al. Controlling attosecond electron dynamics by phase-stabilized polarization gating. Nat Phys, 2006, 2: 319-322

58 Chang Z. Controlling attosecond pulse generation with a double optical gating. Phys Rev A, 2007, 76: 051403

59 Mashiko H, Gilbertson S, Li C, et al. Double optical gating of high-order harmonic generation with carrier-envelope phase stabilized lasers. Phys Rev Lett, 2008, 100: 103906

60 Feng X, Gilbertson S, Mashiko H, et al. Generation of isolated attosecond pulses with 20 to 28 femtosecond lasers. Phys Rev Lett, 2009, 103: 183901

61 Mashiko H, Bell M J, Beck A R, et al. Tunable frequency-controlled isolated attosecond pulses characterized by either $750 \mathrm{~nm}$ or $400 \mathrm{~nm}$ wavelength streak fields. Opt Express, 2010, 18: 25887-25895

62 Vincenti H, Quéré F. Attosecond lighthouses: How to use spatiotemporally coupled light fields to generate isolated attosecond pulses. Phys Rev Lett, 2012, 108: 113904

63 Kim K T, Zhang C, Ruchon T, et al. Photonic streaking of attosecond pulse trains. Nat Photonics, 2013, 7: 651-656

64 Heyl C M, Bengtsson S N, Carlström S, et al. Noncollinear optical gating. New J Phys, 2014, 16: 052001

65 Louisy M, Arnold C L, Miranda M, et al. Gating attosecond pulses in a noncollinear geometry. Optica, 2015, 2: 563

66 Zhong S, He X, Jiang Y, et al. Noncollinear gating for high-flux isolated-attosecond-pulse generation. Phys Rev A, 2016, 93: 033854

67 Tzallas P, Skantzakis E, Kalpouzos C, et al. Generation of intense continuum extreme-ultraviolet radiation by many-cycle laser fields. Nat Phys, 2007, 3: 846-850 
68 Skantzakis E, Tzallas P, Kruse J, et al. Coherent continuum extreme ultraviolet radiation in the sub-100-nJ range generated by a high-power manycycle laser field. Opt Lett, 2009, 34: 1732-1734

69 Timmers H, Sabbar M, Hellwagner J, et al. Polarization-assisted amplitude gating as a route to tunable, high-contrast attosecond pulses. Optica, 2016, 3: 707

70 Chen M C, Mancuso C, Hernandez-Garcia C, et al. Generation of bright isolated attosecond soft X-ray pulses driven by multicycle midinfrared lasers. Proc Natl Acad Sci USA, 2014, 111: E2361-E2367

71 Baltuška A, Udem T, Uiberacker M, et al. Attosecond control of electronic processes by intense light fields. Nature, 2003, 421: 611-615

72 Ye P, He X, Teng H, et al. Full quantum trajectories resolved high-order harmonic generation. Phys Rev Lett, 2014, 113: 073601

73 Hwang S I, Park S B, Mun J, et al. Generation of a single-cycle pulse using a two-stage compressor and its temporal characterization using a tunnelling ionization method. Sci Rep, 2019, 9: 1613

74 Seo M, Tsendsuren K, Mitra S, et al. High-contrast, intense single-cycle pulses from an all thin-solid-plate setup. Opt Lett, 2020, 45: 367

75 Shan B, Ghimire S, Chang Z. Generation of the attosecond extreme ultraviolet supercontinuum by a polarization gating. J Modern Opt, 2005, 52: 277-283

76 Mauritsson J, Johnsson P, Gustafsson E, et al. Attosecond pulse trains generated using two color laser fields. Phys Rev Lett, 2006, 97: 013001

77 Mashiko H, Gilbertson S, Chini M, et al. Extreme ultraviolet supercontinua supporting pulse durations of less than one atomic unit of time. Opt Lett, 2009, 34: 3337

78 Gilbertson S, Wu Y, Khan S D, et al. Isolated attosecond pulse generation using multicycle pulses directly from a laser amplifier. Phys Rev A, 2010, 81: 043810

79 Mashiko H, Oguri K, Sogawa T. Attosecond pulse generation in carbon K-edge region ( $284 \mathrm{eV})$ with sub-250 $\mu \mathrm{J}$ driving laser using generalized double optical gating method. Appl Phys Lett, 2013, 102: 171111

80 Hammond T J, Brown G G, Kim K T, et al. Attosecond pulses measured from the attosecond lighthouse. Nat Photonics, 2016, 10: 171-175

81 Quéré F, Thaury C, Monot P, et al. Coherent wake emission of high-order harmonics from overdense plasmas. Phys Rev Lett, 2006, 96: 125004

82 Lichters R, Meyer-ter-Vehn J, Pukhov A. Short-pulse laser harmonics from oscillating plasma surfaces driven at relativistic intensity. Phys Plasmas, 1996, 3: 3425-3437

83 Pukhov A, an der Brügge D, Kostyukov I. Relativistic laser plasmas for electron acceleration and short wavelength radiation generation. Plasma Phys Controlled Fusion, 2010, 52: 191-223

84 Dromey B, Rykovanov S, Yeung M, et al. Coherent synchrotron emission from electron nanobunches formed in relativistic laser-plasma interactions. Nat Phys, 2012, 8: 804-808

85 Dromey B, Cousens S, Rykovanov S, et al. Coherent synchrotron emission in transmission from ultrathin relativistic laser plasmas. New J Phys, 2013, 15: 015025

86 Wang J W, Zepf M, Rykovanov S G. Intense attosecond pulses carrying orbital angular momentum using laser plasma interactions. Nat Commun, 2019, 10: 5554

87 Li S, Zhang X, Gong W, et al. Spin-to-orbital angular momentum conversion in harmonic generation driven by intense circularly polarized laser. New J Phys, 2020, 22: 013054

88 Zhang Y X, Rykovanov S, Shi M, et al. Giant isolated attosecond pulses from two-color laser-plasma interactions. Phys Rev Lett, 2020, 124: 114802

89 Xu X, Zhang Y, Zhang H, et al. Production of 100-TW single attosecond X-ray pulse. Optica, 2020, 7: 355

90 Kim K T, Villeneuve D M, Corkum P B. Manipulating quantum paths for novel attosecond measurement methods. Nat Photonics, 2014, 8: 187-194

91 Doumy G, Wheeler J, Roedig C, et al. Attosecond synchronization of high-order harmonics from midinfrared drivers. Phys Rev Lett, 2009, 102: 093002

92 Ko D H, Taec Kim K, Nam C H. Attosecond-chirp compensation with material dispersion to produce near transform-limited attosecond pulses. J Phys B-At Mol Opt Phys, 2012, 45: 074015

93 Chang Z. Compensating chirp of attosecond X-ray pulses by a neutral hydrogen gas. OSA Continuum, 2019, 2: 314

94 Lan P F, Lu P X. Generation and control of attosecond laser pulse (in Chinese). Chin Sci Bull, 2021, 66: 847-855 [兰鹏飞, 陆培祥. 阿秒激光脉冲 的产生与操控. 科学通报, 2021, 66: 847-855] 


\title{
Principle and progress of attosecond pulse generation
}

\author{
Zhiyi Wei ${ }^{1,2,3^{*}}$, Siyuan $\mathrm{Xu}^{1,4}$, Yujiao Jiang ${ }^{1,4}$, Yitan Gao ${ }^{1,2}$, Kun Zhao ${ }^{1,3^{*}}$ \& Jiangfeng Zhu ${ }^{4}$ \\ ${ }^{1}$ Beijing National Laboratory for Condensed Matter Physics, Institute of Physics, Chinese Academy of Sciences, Beijing 100190, China; \\ ${ }^{2}$ University of Chinese Academy of Sciences, Beijing 100049, China; \\ ${ }^{3}$ Songshan Lake Material Laboratory, Dongguan 523808, China; \\ ${ }^{4}$ School of Physics and Optoelectronic Engineering, Xidian University, Xi'an 710071, China \\ * Corresponding authors, E-mail: zywei@iphy.ac.cn; zhaokun@iphy.ac.cn
}

With the rapid development of laser technology, the vision of attosecond world has been unfolded. Since the first isolated attosecond pulses produced by high-order harmonic generation in 2001, the time-resolved spectroscopy has entered the attosecond domain and greatly strengthened our understanding of ultrafast electron dynamics in atoms, molecules and condensed matter. Since it approaches the time scale of inner shell electron motion, attosecond pulses become one of the most important tools to explore the electron dynamics. Therefore, developing techniques of attosecond pulse generation is not only essential in optical research field but also imperiously demanded in atomic and molecular physics, which has attracted great attention of researchers worldwide. After years of continuous innovation and breakthroughs, the shortest pulse of 43 attosecond has been achieved. Comparing with the ultrafast laser pulse generation techniques in picosecond or femtosecond regions, the generation of attosecond pulses is rather complex and challenging. From high-order harmonic generation to single attosecond pulse characterization, several crucial technical details need to be considered and investigated carefully, while attosecond gating is a vital link in the chain of technics required by attosecond pulse generation. Up to now, various gating methods have been developed, such as amplitude gating, which, with spectral filtering, takes advantage of the continuous spectrum near the cut-off. In addition, new gating techniques, such as polarization gating, double optical gating, interference polarization gating, polarization-assisted amplitude gating, and phase-matched time gating, also have been proposed or demonstrated to generate isolated attosecond pulses. Recently, spatial gating, which takes advantage of the variation on the driving laser wave front to produce isolated attosecond pulses, has also been developed. Meanwhile, as the intensity at a femtosecond laser focal point now easily exceeds $10^{16} \mathrm{~W} / \mathrm{cm}^{2}$, attosecond pulse generation via laser-plasma interaction has been investigated more intensively. Combined with atto-chirp compensation techniques, these methods are pushing the frontier of ultrafast optics toward shorter and stronger attosecond pulses, and achieving even better time resolution in a broader range of applications. In this article, starting with a general introduction of the principle of attosecond pulse generation, we review various gating techniques for attosecond pulse generation in gas targets, new development of attosecond pulses generated by laser-plasma interaction, as well as atto-chirp compensation for even shorter pulses, which are all crucial for the advancement of attosecond science.

attosecond pulse, attosecond gating technology, high-order harmonics, attosecond pulse compression doi: $10.1360 /$ TB-2020-1525 